OPEN ACCESS

Edited by:

Yuval Gottlieb,

Hebrew University of Jerusalem, Israel

Reviewed by:

Brian Weiss,

Yale University, United States

Volkmar Passoth,

Swedish University of Agricultural

Sciences, Sweden

${ }^{*}$ Correspondence:

Irene Ricci

irene.ricci@unicam.it

Specialty section:

This article was submitted to

Microbial Symbioses,

a section of the journal

Frontiers in Microbiology

Received: 26 October 2020

Accepted: 30 December 2020

Published: 21 January 2021

Citation:

Cappelli A, Favia G and Ricci I (2021)

Wickerhamomyces anomalus in

Mosquitoes: A Promising

Yeast-Based Tool for the

"Symbiotic Control" of

Mosquito-Borne Diseases.

Front. Microbiol. 11:621605.

doi: 10.3389/fmicb.2020.621605

\section{Wickerhamomyces anomalus in Mosquitoes: A Promising Yeast-Based Tool for the "Symbiotic Control" of Mosquito-Borne Diseases}

\author{
Alessia Cappelli, Guido Favia and Irene Ricci* \\ School of Biosciences and Veterinary Medicine, University of Camerino, CIRM Italian Malaria Network, Camerino, Italy
}

The ascomycete yeast Wickerhamomyces anomalus is a mutualistic symbiont of different insects, including diptera vectors of diseases. Although fungal symbioses have been so far poorly characterized, the topic is gaining attention as yeast-insect interactions can provide pivotal information on insect biology, such as their environmental adaptation or vectorial capability. We review the symbiosis between $W$. anomalus and mosquitoes, which implies nutritional and protective functions. Furthermore, we focus on antiplasmodial effects of $W$. anomalus in malaria vectors and discuss the yeast potential for the "symbiotic control" (SC) of mosquito-borne diseases (MBDs).

Keywords: Wickerhamomyces anomalus, yeast, insect, mosquito, malaria, symbiosis, mycobiota, symbiotic control

\section{INTRODUCTION}

Mosquitoes comprise different species that are vectors of pathogens to humans and other animals. The most important mosquito-borne diseases (MBDs) are transmitted by three mosquito genera: Aedes, Anopheles, and Culex. Aedes transmit several arboviruses including Dengue, Yellow Fever, Chikungunya, Zika, and West Nile; Anopheles spread malaria parasites (Plasmodium spp.); Culex is a major vector of West Nile virus and filarial nematodes. In the last decades, climate changes, anthropization of new habitats, and international trade have favored the global expansion of mosquito vectors and emergence of MBDs causes major public health concerns in new geographic areas (Brugueras et al., 2020). Since vaccines against most mosquito-borne pathogens are not available, their prevention relies mainly on insecticides. However, insecticides become less effective as vectors develop resistance and the overuse of chemical insecticides increases the costs besides having deleterious effects on non-target species and the environment. Thus, innovative eco-friendly alternatives are requested. New control methods are focusing on the rising knowledge of the mosquito microbial community (microbiota) and its effect on the mosquito-pathogen relationship. Understanding the biology of symbionts in insect vectors is essential for the development of biological control strategies like the "symbiotic control" (SC; Bourtzis et al., 2014). The SC is a promising non-chemical method for the control of vectors and diseases they transmit. This approach exploits symbiotic microorganisms, bacteria or fungi, with the aim of reducing the vector capability (Niang et al., 2018). One of the strategies 
for the SC of vector-borne pathogens is based on the exploitation of antagonism between symbionts and pathogens, as in the case of the bacteria Wolbachia that is applied for the control of mosquito-borne viruses (Frentiu, 2017).

The analysis of the mosquito-associated microbiota (bacteria, fungi, protists, viruses, and nematodes) is gaining attention since microbes are recognized to influence many traits of the mosquito biology, such as development, physiology, immunity, and vector competence (Guégan et al., 2018). While an increasing number of studies have focused on bacteria, the fungal community (mycobiota) has been largely neglected, but recent studies show the presence of an important fungal diversity in mosquitoes (Malassigné et al., 2020). Mosquito-mycobiota is mainly composed of Ascomycota, which comprise mostly species of Pezizomycotina and Saccharomycotina (subphyla); Pezizomycotina include species of filamentous fungi, whereas Saccharomycotina are basically represented by yeasts, such as Candida, Meyerozyma, Pichia, and Wickerhamomyces, that adapt to survive in the insect gut and different mutualistic yeast-insect symbioses based on trophic interdependence have been described (Malassigné et al., 2020). Yeasts generate signals of sugar resources through metabolic pathways that produce compounds, such as fermentative volatiles, that attract insects. On the other hand, insects disperse yeasts over a variety of sugar sources and provide them with food and a habitat (the gut) to mate or generate sexual forms, increasing yeast biodiversity (Madden et al., 2018). Yeasts are important not only for attraction to food, they influence oviposition sites and larval development, but also supply diet integration of adults providing organic nitrogen, essential vitamins, and lipids (Stefanini, 2018). In summary, insects are an essential component of the ecology of ascomycetous yeasts, and the latter influence many traits of the insects' biology.

Wickerhamomyces anomalus (class: saccharomycetes) is a budding yeast identified in the gut of diverse insects, in which it is suggested as a mutualist symbiont with nutritional and protective functions (Toki et al., 2013; Steyn et al., 2016). The presence of $W$. anomalus is reported in different orders of insects, such as mosquitoes (Diptera: Culicidae), sand flies (Diptera: Psychodidae), honey bees (Hymenoptera), plant hoppers (Hemiptera), and beetles (Coleoptera). Several studies focused on the yeast association with mosquitoes, in which W. anomalus is identified in Anopheles, Aedes, and Culex species (Ricci et al., 2011a; Muturi et al., 2016; Steyn et al., 2016). Interestingly, the strain of $W$. anomalus identified in the malaria vector Anopheles stephensi produces a killer toxin (KT) with wide antimicrobial activities that may protect the host from entomopathogenic microbes (Ricci et al., 2011b). KTs are glycoproteins with exo- $\beta$-1,3-glucanase enzymatic activity that hydrolyze glucans on the surface of different microbial targets, such as viruses, bacteria, fungi, and protozoa (Walker, 2011). Additional studies demonstrated a strong KT-based effect against the protozoa Plasmodium berghei that prevent the parasite development in the midgut of An. stephensi (Cappelli et al., 2019). Here, we review the $W$. anomalus symbiotic association with mosquitoes and focus on the antiplasmodial effect, discussing the potential application of a new yeast-based approach for the SC of malaria and possibly other MBDs.

\section{GENERAL FEATURES OF Wickerhamomyces anomalus}

Wickerhamomyces anomalus, formerly Pichia anomala and Hansenula anomala, was renamed following phylogenetic studies (Daniel et al., 2012). The biotechnological potential of this yeast and its use in different industrial applications derive from capability of $W$. anomalus to secrete antimicrobials factors acting on a broad spectrum of pathogens (Walker, 2011). In the last decade, the yeast has drawn special attention and the genome sequences of two environmental strains have been published (Schneider et al., 2012; Cunha et al., 2020). The European Food Safety Authority (EFSA) classifies $W$. anomalus at biosafety level one and different "killer strains" (yeast isolates that produce KTs) are used as biocontrol agents with inhibitory effects against mold and bacteria in the agro-food sector (Sundh and Melin, 2011). Specific properties make $W$. anomalus a suitable product for different biotechnological applications: (i) adaptation to a wide range of growth conditions in terms of temperature $\left(3-37^{\circ} \mathrm{C}\right), \mathrm{pH}$ value (2-12), and osmolarity; (ii) robustness and competitiveness in different habitats; (iii) ability to produce biomass on a large scale; (iv) stress-tolerance, survival, and maintenance of biocontrol activity in different formulations and after desiccation (Passoth et al., 2011).

Wickerhamomyces anomalus has been isolated from different environmental matrices: flowers and leaves, food and feed systems, and waters and insects (Walker, 2011). Moreover, opportunistic strains have been detected in immunocompromised patients and, even though few outbreaks have been reported, they should be taken into consideration (Dutra et al., 2020). Thus, distinguishing pathogenic from non-pathogenic strains is crucial for future applications of $W$. anomalus. Since it was identified in hematophagous insects (mosquitoes and sand flies), a study investigated whether $W$. anomalus infects humans: screening immunocompromised and malaria patients, and healthy volunteers (exposed to mosquito bites) revealed that $W$. anomalus is not relevant to human, consistently with the rare reports of fungemia (Epis et al., 2015). Safety tests showed that W. anomalus does not harm mammalian cells, suggesting that they are not sensible to the yeast killer activity (Cappelli et al., 2019).

\section{Wickerhamomyces anomalus-MOSQUITO SYMBIOSES AND THEIR IMPACT ON THE INSECT BIOLOGY}

On the background that $W$. anomalus has been isolated from different environmental matrices such as flowers and water, mosquitoes larvae can acquire the yeast from the aquatic breeding sites, whereas adults by feeding on nectars. First identifications of $W$. anomalus in mosquitoes were in Anopheles stephensi (lab-reared colony), and Anopheles gambiae, 
Aedes aegypti, and Aedes albopictus (lab-reared colonies and wild samples; Ricci et al., 2011a,b). An in-depth screening of the mycobiota in Culex spp. revealed the presence of W. anomalus in Culex pipens (wild samples; Steyn et al., 2016). More recent investigations by metagenomic analyses of invasive species identified the yeast in Aedes japonicus and Aedes triseriatus (wild samples; Muturi et al., 2016). However, mosquitoes are not the only host of $W$. anomalus since it is found and suggested as mutualist symbiont in Phlebotomus perniciosus (sand fly), Apis mellifera (honeybee), Laodelphax striatellatus (planthopper), and Doubledaya bucculenta (beetle; Table 1).

\section{Transmission Routes and Influence on Larval Development}

Investigations in An. stephensi revealed the presence of $W$. anomalus in all the developmental stages of the mosquito (larvae, pupae, and adults). Wickerhamomyces anomalus is supposed to be associated with pre-adult stages also in Ae. japonicus and Ae. triseriatus, since it has been isolated from the midgut of adult females hatched in the laboratory from wild-caught pupae. Different results have been reported in Cx. pipens, which seems to host $W$. anomalus only at the larval stages, in fact, adult mosquitoes caught near the larval breeding sites tested negative for the yeast detection. As observed for most bacteria, structure and abundance of fungal communities vary during the mosquito life-cycle with a significant reduction of fungal diversity in the midgut of newly emerged adults (Steyn et al., 2016) as well as in females after blood ingestion (Muturi et al., 2016). Conversely, the presence of $W$. anomalus in newly emerged adults reared under controlled conditions supports the hypothesis of a transstadial transmission in An. stephensi, Ae. japonicus, and Ae. triseriatus. Except for the observations reported in Cx. pipiens, $W$. anomalus bypasses the fungal reduction during the mosquito metamorphosis from larva to adult, in different species. This is a prerogative of few microbes, which implies robustness and competitiveness in different habitats, since the pupal gut meet physiological adaptations that are needed for molting as hypoxia (Valzania et al., 2018).

Wickerhamomyces anomalus has been detected both in the midgut and gonads (male and female) of adults An. stephensi, suggesting its possible involvement in the mosquito reproduction and vertical transmission routes. Indeed, experiments performed using a monoclonal antibody targeting KTs demonstrated that W. anomalus is not affected by the blood meal and it is transmitted to the offspring (Cappelli et al., 2014). Wickerhamomyces anomalus maternal transmission through the egg surface in newly hatched larvae has been shown in the non-social beetle $D$. bucculenta, whose eggs acquire the yeast from the ovipositor-associated fungal pocket (mycangium) of adult females (Toki et al., 2013). This mechanism ensures an immediate acquisition of $W$. anomalus that is an essential food source for the larval development of D. bucculenta. A similar mutualism is proposed also in the planthopper L. striatellatus, in which W. anomalus favors the larval development and localizes the fat body of adult females, like other symbionts that are transmitted to the progeny through the ovary (Cao et al., 2015). Concerning mosquitoes, a positive

TABLE 1 | Wickerhamomyces anomalus in mosquitoes and other insects.

\begin{tabular}{|c|c|c|c|c|}
\hline Insect host (order) & Species & $\begin{array}{l}\text { Symbiotic functions } \\
\text { described (bold) or } \\
\text { proposed (?) }\end{array}$ & $\begin{array}{l}\text { Developmental stages } \\
\text { (organs) }\end{array}$ & References \\
\hline \multirow{7}{*}{ Mosquito (Diptera: Culicidae) } & $\begin{array}{l}\text { Anopheles stephensi } \\
\text { (laboratory reared colonies) }\end{array}$ & $\begin{array}{l}\text { Protective role: killer strain } \\
\text { with antifungal and } \\
\text { antiplasmodial effects } \\
(\beta-1,3-\text { glucanase activity); } \\
\text { Nutritional role in larvae and } \\
\text { adults (?) }\end{array}$ & $\begin{array}{l}\text { Larvae, pupae, adult male, and } \\
\text { female (midgut, gonads) }\end{array}$ & $\begin{array}{l}\text { Ricci et al., 2011b; Cappelli } \\
\text { et al., 2014, 2019; Valzano } \\
\text { et al., 2016; Cecarini et al., } \\
2019\end{array}$ \\
\hline & $\begin{array}{l}\text { Anopheles gambiae (wild } \\
\text { samples and laboratory reared } \\
\text { colonies) }\end{array}$ & Nutritional/protective role (?) & Adult male and female & Ricci et al., $2011 a$ \\
\hline & $\begin{array}{l}\text { Aedes albopictus (wild samples } \\
\text { and laboratory reared colonies) }\end{array}$ & Nutritional/protective role (?) & Adult male and female & Ricci et al., 2011a \\
\hline & $\begin{array}{l}\text { Aedes aegypti (wild samples } \\
\text { and laboratory reared colonies) }\end{array}$ & Nutritional/protective role (?) & Adult male and female & Ricci et al., 2011a \\
\hline & Aedes japonicus (wild samples) & Nutritional/protective role (?) & Adult female (midgut) & Muturi et al., 2016 \\
\hline & Aedes triseriatus (wild samples) & Nutritional/protective role (?) & Adult female (midgut) & Muturi et al., 2016 \\
\hline & Culex pipiens (wild samples) & $\begin{array}{l}\text { Nutritional role: larval } \\
\text { development; protective role (?) }\end{array}$ & Larvae & Steyn et al., 2016 \\
\hline \multirow[t]{2}{*}{ Sand fly (Diptera: Psychodidae) } & Phlebotomus perniciosus & $\begin{array}{l}\text { Protective role: killer strain } \\
\text { with antifungal effect; } \\
\text { nutritional role (?) }\end{array}$ & $\begin{array}{l}\text { Larvae (gut), adult male and } \\
\text { female }\end{array}$ & $\begin{array}{l}\text { Martin et al., 2016; Giovati et al., } \\
2018\end{array}$ \\
\hline & & Protective role: & & \\
\hline Honeybee (Hymenoptera) & Apis mellifera & $\begin{array}{l}\text { immunomodulatory effect; } \\
\text { nutritional role (?) }\end{array}$ & Adult female (midgut) & Tauber et al., 2019 \\
\hline Plant hopper (Hemiptera) & Laodelphax striatellus & $\begin{array}{l}\text { Nutritional role: larval } \\
\text { development; protective role (?) }\end{array}$ & $\begin{array}{l}\text { Nymphs, adult female (fat } \\
\text { body) }\end{array}$ & Cao et al., 2015 \\
\hline Beetle (Coleoptera) & Doubledaya bucculenta & $\begin{array}{l}\text { Nutritional role: larval } \\
\text { development; protective role (?) }\end{array}$ & $\begin{array}{l}\text { Eggs, larvae, and adult female } \\
\text { (putative mygangium) }\end{array}$ & Toki et al., 2012, 2013 \\
\hline
\end{tabular}


influence of $W$. anomalus on larval survival and pupation has been shown in Cx. pipiens, using the yeast as an exclusive food source (Steyn et al., 2016).

\section{Role in Digestive Processes of Adult Insects}

In adult mosquitoes, $W$. anomalus is supposed to establish trophic interactions useful to integrate a sugar restricted diet. This support has been described in L. striatellatus, in which $W$. anomalus provides nutrients to compensate the adult planthopper diet for the unbalanced composition of amino acids in plant phloem (Cao et al., 2015). Additionally, as floral nectarresiding yeast $W$. anomalus may support nutritional functions by acting on sugar digestibility. For instance, it may participate by fermentation to the breakdown of sugar while still residing on the flowers, as well as in the insect gut after the nectar collection, as suggested in A. mellifera (Tauber et al., 2019).

Another contribution of $W$. anomalus in digestive processes may be specifically exerted in adult female mosquitoes after the blood ingestion, since the yeast is among the few microbes that persist in the midgut after blood ingestion (Cappelli et al., 2019). This peculiarity is likely due to the ability of $W$. anomalus to adapt to different growth conditions, as a wide range of $\mathrm{pH}$ values and osmolarity. Indeed, chemical setup in the female midgut sensibly varies depending on the meal (sugar or blood), and during the blood digestion it changes from acid to basic pH values (del Pilar Corena et al., 2005). Moreover, W. anomalus withstands high concentrations of uric acid likely implying complete degradation pathways, thus it has potential to participate in the urate degradation and in the removal of nitrogenous wastes deriving from the blood digestion. Martin and collaborators demonstrated this contribution in females of the haematophagous diptera $P$. perniciosus by another insectassociated yeast, Meyerozyma guilliermondii (Martin et al., 2018).

\section{Antimicrobial Properties and Defense of the Host}

In addition to nutritional support for larvae and adults, $W$. anomalus has been suggested for protective functions thanks to antimicrobial properties. It is demonstrated that yeasts can selectively shape the insect microbial community inhibiting the development of entomopathogenic fungi and increasing the development of mutualistic fungi (Davis et al., 2011). For example, $W$. anomalus defends larva-inhabiting bamboo internodes of $D$. bucculenta from the invasion of microbial contaminants, so that the inner surface of larval sites is covered with a fungal layer that represents a $W$. anomalus monoculture (Toki et al., 2012). However, the molecular basis of the antimicrobial activity in D. bucculenta has not yet been clarified. Instead, protective functions based on killer activities have been proposed in mosquitoes and the related mechanisms of action have been characterized (Cappelli et al., 2014). A strain of W. anomalus (F17.12) isolated from An. stephensi produces a KT with a strong antimicrobial activity likely exerting defense from entomopathogens. F17.12 secretes KT in specific cultural conditions as well as in vivo in the mosquito body, both in midguts and gonads (female and male). The KT-antimicrobial activity has been demonstrated in vitro against different susceptible yeast strains (e.g., of Candida albicans) and at diverse acidity conditions. Indeed, F17.12 KT activity assays showed that the yeast killer property spans a wide range of $\mathrm{pH}$ values (4.5-8). This finding fits well possible antimicrobial competition of $W$. anomalus in the mosquito body, since different physiological conditions occur in different organs (midgut and gonads). Likely in mosquitoes, a killer strain of $W$. anomalus displaying a killer phenotype and candidacidal activity has been isolated also in larvae and adults (male and female) of the sand fly P. pernicious (Martin et al., 2016; Giovati et al., 2018).

\section{INFLUENCE OF Wickerhamomyces anomalus ON THE MOSQUITO' VECTOR COMPETENCE}

\section{Antiplasmodial Effects}

On the basis that $W$. anomalus releases active $\mathrm{KT}$ in the female mosquito's midgut even after the blood meal, the possible killer effect against the parasite in the mosquito midgut (early sporogonic stages) has been investigated. A strong antiplasmodial effect of KT has been demonstrated in vitro against early sporogonic stages (ookinetes) of the malaria rodent parasite P. berghei, with an inhibition of the parasite survival by $90 \%$, and several morphological/structural alterations revealed by microscopy analysis (Valzano et al., 2016). These findings prompted additional in vivo studies highlighting that $W$. anomalus inhibits the development of $P$. berghei in the midgut of the malaria vector An. stephensi (Cappelli et al., 2019). Dietary supplementation of adult females with F17.12 reduced ookinetes in the midgut by $65 \%$. In vitro and in vivo inhibition rates cannot be compared because of substantial differences between the two experimental systems. However, the result obtained in vivo is very important considering that the number of ookinetes that complete their development in malaria experimental models (e.g., An. stephensi/P. berghei) is considerably higher than those observed in wild mosquitoes (100-folder plus; Sinden, 1997). Therefore, $W$. anomalus has potential to significantly reduce the vector capability of mosquitoes in the field. Likely in mosquitoes, the yeast may also affect Leishmania (protozoan parasite) and phleboviruses in P. perniciosus, though further studies are necessary to evaluate anti-pathogen effects in the sand fly.

\section{Direct and Indirect Mechanisms of Action}

Valzano et al. (2016) showed that effects on P. berghei ookinetes are due to direct implication of the KT-enzymatic activity. In particular, treatment with castanospermine, which is an inhibitor of exo- $\beta-1,3$-glucanase-mediated activities, reduced the killer effect on the parasite and the membrane damage. These data suggest that $P$. berghei death is induced by the hydrolysis of $\beta$-glucans located in the cell-wall of the parasite. The biochemical characterization of the KT purified from F17.12 identified a glycoprotein of $140 \mathrm{kD}$ and limited electrophoretic mobility, 
corresponding to a high molecular weight $\beta$-glucosidase, as confirmed by activity tests in presence of castanospermine and the analysis of the predictive three-dimensional structure (Cecarini et al., 2019). Besides direct killer effects, yeasts can act by indirect mechanisms modulating the host immune system. For instance, $W$. anomalus has been reported as an active component of the gut with immunomodulatory effects in A. mellifera (Tauber et al., 2019). As regard mosquitoes, interesting results have been obtained by a dietary supplementation of An. stephensi with a KT non-producer strain of $W$. anomalus (UM3; Cappelli et al., 2019). The study demonstrated a reduction of the intensity of $P$. berghei infection by $30 \%$ (around half that by F17.12). Since UM3 is not able to induce any effect in vitro on cultivated parasites, indirect effects on the mosquito immune response are supposed. By the combination of direct KT-mediated activity and immune modulation of the host system, W. anomalus may sensibly affect the mosquito vector competence.

\section{Wickerhamomyces anomalus IS A PROMISING TOOL FOR "SYMBIOTIC CONTROL" OF MBDs}

Among a wide number of mosquito-associated yeasts (Malassigné et al., 2020), $W$. anomalus is a promising candidate for SC strategies against malaria and possibly other MBDs. We summarize main features that support this finding: (i) it is a mutualistic symbiont in Anopheles, Aedes, and Culex mosquitoes (impling a stable association with vectors); (ii) it bypasses the fungal reduction during the mosquito metamorphosis and after the blood ingestion; (iii) it spreads by trans-stadial and vertical transmission routes (impling selfspreading among mosquitoes and reducing efforts and costs of delivery); (iv) it inhibits the malaria parasite in the vector mosquitoes suggesting that, thanks to the wide killer activity, it has potential to affect a broad spectrum of mosquito-borne pathogens (e.g., arboviruses); (v) it is considered safe for humans and the environment (EFSA authorizes use of killer strains);

\section{REFERENCES}

Bourtzis, K., Dobson, S. L., Xi, Z., Rasgon, J. L., Calvitti, M., Moreira, L. A., et al. (2014). Harnessing mosquito-Wolbachia symbiosis for vector and disease control. Acta Trop. 132, S150-S163. doi: 10.1016/j.actatropica.2013.11.004

Brugueras, S., Fernández-Martínez, B., Martínez-de la Puente, J., Figuerola, J., Porro, T. M., Rius, C., et al. (2020). Environmental drivers, climate change and emergent diseases transmitted by mosquitoes and their vectors in southern Europe: a systematic review. Environ. Res. 191:110038. doi: 10.1016/j. envres.2020.110038

Cao, W., Ma, Z., Chen, Y. H., and Yu, X. (2015). Pichia anomala, a new species of yeast-like endosymbionts and its variation in small brown planthopper (Laodelphax striatellus). J. Biosci. Bioeng. 119, 669-673. doi: 10.1016/j.jbiosc.2014.11.007

Cappelli, A., Ulissi, U., Valzano, M., Damiani, C., Epis, S., Gabrielli, M. G., et al. (2014). A Wickerhamomyces anomalus killer strain in the malaria vector Anopheles stephensi. PLoS One 9:e95988. doi: 10.1371/journal.pone.0095988

Cappelli, A., Valzano, M., Cecarini, V., Bozic, J., Rossi, P., Mensah, P., et al. (2019). Killer yeasts exert anti-plasmodial activities against the malaria (vi) it is clinically not relevant and its killer effect is based on exo- $\beta-1,3$-glucanase enzymatic activities (which targets microbes but is harmless in mammalian cell); (vii) it can be ingested by larvae and adults as a food source (advantage of yeasts over intracellular symbionts such as Wolbachia); and (viii) it can be formulated at low costs of production and released in the environment by dried-yeasts containing tablets, as recently carried out with fungal-larvicides used against Aedes spp. (Stewart et al., 2020).

\section{CONCLUDING REMARKS}

Evidence of the ability of $W$. anomalus to inhibit the malaria parasite multiplication and survival in the mosquito, pose the bases for an exploitation of this killer yeast as an environmental-friendly and safe tool to interfere with vector competence. Since $W$. anomalus has been isolated in different vector species, further investigations are worthy for understanding whether it is able to interfere with different mosquito-borne pathogens. Its biological characteristics and the possibility of a large scale manifacture of a practical product both for storage and release, make $W$. anomalus a new yeast-based tool for "symbiotic control" strategies of MBDs.

\section{AUTHOR CONTRIBUTIONS}

AC and IR: conceptualization, original draft preparation, and editing. GF: manuscript reviewing. IR: project administration and supervision. All authors contributed to the article and approved the submitted version.

\section{FUNDING}

The work was supported by the European Union Horizon 2020 under Grant Agreement No. 842429 to IR. parasite Plasmodium berghei in the vector mosquito Anopheles stephensi and in mice. Parasit. Vectors 12:329. doi: 10.1186/s13071-019-3587-4

Cecarini, V., Cuccioloni, M., Bonfili, L., Ricciutelli, M., Valzano, M., Cappelli, A., et al. (2019). Identification of a killer toxin from Wickerhamomyces anomalus with $\beta$-glucanase activity. Toxins 11:568. doi: 10.3390/toxins 11100568

Cunha, A. C., Santos, R. A. C. D., Riaño-Pachon, D. M., Squina, F. M., Oliveira, J. V. C., Goldman, G. H., et al. (2020). Draft genome sequence of Wickerhamomyces anomalus LBCM1105, isolated from cachaça fermentation. Genet. Mol. Biol. 43:e20190122. doi: 10.1590/1678-4685GMB-2019-0122

Daniel, H. -M., Redhead, S. A., Schnurer, J., Naumov, G. I., and Kurtzman, C. P. (2012). (2049-2050) proposals to conserve the name Wickerhamomyces against Hansenula and to reject the name Saccharomyces sphaericus (Ascomycota: Saccharomycotina). Taxon 61, 459-461. doi: 10.1002/tax.612015

Davis, T. S., Hofstetter, R. W., Foster, J. T., Foote, N. E., and Keim, P. (2011). Interactions between the yeast Ogataea pini and filamentous fungi associated with the western pine beetle. Microb. Ecol. 61, 626-634. doi: 10.1007/ s00248-010-9773-8 
del Pilar Corena, M., VanEkeris, L., Salazar, M. I., Bowers, D., Fiedler, M. M., Silverman, D., et al. (2005). Carbonic anhydrase in the adult mosquito midgut. J. Exp. Biol. 208, 3263-3273. doi: 10.1242/jeb.01739

Dutra, V. R., Silva, L. F., Oliveira, A. N. M., Beirigo, E. F., Arthur, V. M., Bernardes da Silva, R., et al. (2020). Fatal case of fungemia by Wickerhamomyces anomalus in a pediatric patient diagnosed in a teaching hospital from Brazil. J. Fungi 6:E147. doi: 10.3390/jof6030147

Epis, S., Capone, A., Martin, E., Paolucci, M., Bazzocchi, C., Valzano, M., et al. (2015). A rapid qPCR method to investigate the circulation of the yeast Wickerhamomyces anomalus in humans. New Microbiol. 38, 577-581.

Frentiu, F. D. (2017). Lipids and pathogen blocking by Wolbachia. Trends Parasitol. 33, 916-917. doi: 10.1016/j.pt.2017.10.007

Giovati, L., Santinoli, C., Ferrari, E., Ciociola, T., Martin, E., Bandi, C., et al. (2018). Candidacidal activity of a novel killer toxin from Wickerhamomyces anomalus against fluconazole-susceptible and resistant strains. Toxins 10:68. doi: $10.3390 /$ toxins 10020068

Guégan, M., Zouache, K., Démichel, C., Minard, G., Tran Van, V., Potier, P., et al. (2018). The mosquito holobiont: fresh insight into mosquito-microbiota interactions. Microbiome 6:49. doi: 10.1186/s40168-018-0435-2

Madden, A. A., Epps, M. J., Fukami, T., Irwin, R. E., Sheppard, J., Sorger, D. M., et al. (2018). The ecology of insect-yeast relationships and its relevance to human industry. Proc. Biol. Sci. 285:20172733. doi: 10.1098/rspb.2017.2733

Malassigné, S., Valiente Moro, C., and Luis, P. (2020). Mosquito mycobiota: an overview of non-entomopathogenic fungal interactions. Pathogens 9:564. doi: 10.3390/pathogens9070564

Martin, E., Bongiorno, G., Giovati, L., Montagna, M., Crotti, E., Damiani, C., et al. (2016). Isolation of a Wickerhamomyces anomalus yeast strain from the sandfly Phlebotomus perniciosus displaying the killer phenotype. Med. Vet. Entomol. 30, 101-106. doi: 10.1111/mve.12149

Martin, E., Varotto Boccazzi, I., De Marco, L., Bongiorno, G., Montagna, M., Sacchi, L., et al. (2018). The mycobiota of the sand fly Phlebotomus perniciosus: involvement of yeast symbionts in uric acid metabolism. Environ. Microbiol. 20, 1064-1077. doi: 10.1111/1462-2920.14044

Muturi, E. J., Bara, J. J., Rooney, A. P., and Hansen, A. K. (2016). Midgut fungal and bacterial microbiota of Aedes triseriatus and Aedes japonicus shift in response to La Crosse virus infection. Mol. Ecol. 25, 4075-4090. doi: 10.1111/mec.13741

Niang, E. H. A., Bassene, H., Fenollar, F., and Mediannikov, O. (2018). Biological control of mosquito-borne diseases: the potential of Wolbachia-based interventions in an IVM framework. J. Trop. Med. 2018:1470459. doi: 10.1155/2018/1470459

Passoth, V., Olstorpe, M., and Schnürer, J. (2011). Past, present and future research directions with Pichia anomala. Antonie Van Leeuwenhoek 99, 121-125. doi: 10.1007/s10482-010-9508-3.

Ricci, I., Damiani, C., Scuppa, P., Mosca, M., Crotti, E., Rossi, P., et al. (2011b). The yeast Wickerhamomyces anomalus (Pichia anomala) inhabits the midgut and reproductive system of the Asian malaria vector Anopheles stephensi. Environ. Microbiol. 13, 911-921. doi: 10.1111/j.1462-2920.2010.02395.x

Ricci, I., Mosca, M., Valzano, M., Damiani, C., Scuppa, P., Rossi, P., et al. (2011a). Different mosquito species host Wickerhamomyces anomalus (Pichia anomala): perspectives on vector-borne diseases symbiotic control. Antonie Van Leeuwenhoek 99, 43-50. doi: 10.1007/s10482-010-9532-3
Schneider, J., Rupp, O., Trost, E., Jaenicke, S., Passoth, V., Goesmann, A., et al. (2012). Genome sequence of Wickerhamomyces anomalus DSM 6766 reveals genetic basis of biotechnologically important antimicrobial activities. FEMS Yeast Res. 12, 382-386. doi: 10.1111/j.1567-1364.2012.00791.x

Sinden, R. E. (1997). "Infection of mosquitoes with rodent malaria" in Molecular biology of insect disease vectors: A methods manual. eds. J. M. Crampton, C. B. Beard and C. Louis (Dordrecht: Springer), 67-91.

Stefanini, I. (2018). Yeast-insect associations: it takes guts. Yeast 35, 315-330. doi: 10.1002/yea.3309

Stewart, A. T. M., Winter, N., Igiede, J., Hapairai, L. K., James, L. D., Feng, R. S., et al. (2020). Community acceptance of yeast interfering RNA larvicide technology for control of Aedes mosquitoes in Trinidad. PLoS One 15:e0237675. doi: 10.1371/journal.pone.0237675

Steyn, A., Roets, F., and Botha, A. (2016). Yeasts associated with Culex pipiens and Culex theileri mosquito larvae and the effect of selected yeast strains on the ontogeny of Culex pipiens. Microb. Ecol. 71, 747-760. doi: 10.1007/ s00248-015-0709-1

Sundh, I., and Melin, P. (2011). Safety and regulation of yeasts used for biocontrol or biopreservation in the food or feed chain. Antonie Van Leeuwenhoek 99, 113-119. doi: 10.1007/s10482-010-9528-z

Tauber, J. P., Nguyen, V., Lopez, D., and Evans, J. D. (2019). Effects of a resident yeast from the honeybee gut on immunity, microbiota and Nosema disease. Insects 10:296. doi: 10.3390/insects10090296

Toki, W., Takahashi, Y., and Togashi, K. (2013). Fungal garden making inside bamboos by a non-social fungus-growing beetle. PLoS One 8:e79515. doi: 10.1371/journal.pone.0079515

Toki, W., Tanahashi, M., Togashi, K., and Fukatsu, T. (2012). Fungal farming in a non-social beetle. PLoS One 7:e41893. doi: 10.1371/journal.pone.0041893

Valzania, L., Coon, K. L., Vogel, K. J., Brown, M. R., and Strand, M. R. (2018) Hypoxia-induced transcription factor signaling is essential for larval growth of the mosquito Aedes aegypti. Proc. Natl. Acad. Sci. U. S. A. 115, 457-465. doi: 10.1073/pnas.1719063115

Valzano, M., Cecarini, V., Cappelli, A., Capone, A., Bozic, J., Cuccioloni, M., et al. (2016). A yeast strain associated to Anopheles mosquitoes produces a toxin able to kill malaria parasites. Malar. J. 15:21. doi: 10.1186/ s12936-015-1059-7

Walker, G. M. (2011). Pichia anomala: cell physiology and biotechnology relative to other yeasts. Antonie Van Leeuwenhoek 99, 25-34. doi: 10.1007/ s10482-010-9491-8

Conflict of Interest: The authors declare that the research was conducted in the absence of any commercial or financial relationships that could be construed as a potential conflict of interest.

Copyright (C) 2021 Cappelli, Favia and Ricci. This is an open-access article distributed under the terms of the Creative Commons Attribution License (CC BY). The use, distribution or reproduction in other forums is permitted, provided the original author(s) and the copyright owner(s) are credited and that the original publication in this journal is cited, in accordance with accepted academic practice. No use, distribution or reproduction is permitted which does not comply with these terms. 